\title{
Suspended Nanoporous Membranes as Interfaces for Neuronal Biohybrid Systems
}

\author{
Bernhard Wolfrum, ${ }^{*}$ Yulia Mourzina, Frank Sommerhage, and \\ Andreas Offenhăusser
}

ISG2: Institute for Thin Films and Interfaces, Center of Nanoelectronic Systems for Information Technology, Research Center Juelich, 52425 Juelich, Germany

Received December 1, 2005; Revised Manuscript Received January 17, 2006

\begin{abstract}
A biohybrid system composed of neuronal cells and silicon-supported nanoporous membranes has been designed to facilitate control of the biochemical environment of neuronal networks with cellular resolution. The membranes may exhibit variable pore sizes and interpore distances and are interfaced to a microfluidic device. Different porosity parameters give rise to changes in the transconductance of the nanopores and can therefore be used to control diffusion of molecules through the membranes. It was shown that the porous membranes are biocompatible with primary vertebrate as well as insect neurons. Our results indicate that nanoporous membranes may be used to interface with biological materials in a biohybrid system, for example as an artificial chemical synapse interface.
\end{abstract}

With the development of biosensors and neuroprosthetic devices, biohybrid systems are starting to gain a lot of attention. ${ }^{1}$ Important aspects for many cell-based biohybrid systems are the biocompatibility of the interfacing substrate as well as the functional coupling between biological and artificial materials. Nanoporous materials offer new possibilities as biointerfaces and may be used in biomedical applications. ${ }^{2}$ For example nanoporous alumina promises to be advantageous in implants and has already been shown to be biocompatible with bone cells. ${ }^{3}$ Recently, Takoh et al. ${ }^{4}$ have demonstrated the use of nanoporous alumina membranes as an interface in localized chemical drug delivery for HeLa cells in culture. Herein we show, that porous alumina membranes may also be used to provide a chipbased nanostructured interface to address neuronal cells with high resolution. However, the brittle nature of suspended porous alumina membranes and their weakness toward certain chemicals are disadvantageous, especially regarding their reusability. Therefore we transferred the alumina nanopores onto silicon nitride/silicon oxide to create stable suspended nanoporous $\mathrm{Si}_{3} \mathrm{~N}_{4} / \mathrm{SiO}_{2}$ membranes, which can be cleaned and reused after cell experiments. The suspended nanoporous membrane patches are processed on a silicon wafer. The silicon substrate acts as a support and facilitates the integration of further stimulation or sensing functionalities using standard semiconductor technologies. In addition microfluidics can be integrated into the system allowing for localized chemical stimulation of cells.

The process for the fabrication of suspended nanoporous membranes on a silicon support is based on anisotropic chemical silicon etching from the backside of the wafer in combination with anodization of thin aluminum films. ${ }^{5-7} \mathrm{~A}$ sketch of the fabrication process is shown in Figure 1.

In the first preparation step of the microfluidic structures, double-sided polished $\langle 100\rangle$-oriented silicon wafers with a thickness of $335-365 \mu \mathrm{m}$ and a resistance of $1-10 \Omega \mathrm{cm}$ were oxidized under wet conditions at $1100{ }^{\circ} \mathrm{C}$ to grow 400 $\mathrm{nm}$ silicon oxide. Afterward, a $130 \mathrm{~nm}$ film of silicon nitride was deposited onto the silicon oxide by low-pressure chemical vapor deposition. The backside of the wafer was patterned using lithography combined with reactive ion etching (RIE). Then the patterned structures were etched anisotropically in $20 \%$ potassium hydroxide solution at 85 ${ }^{\circ} \mathrm{C}$. This leads to truncated pyramids where the etched region is confined by the $\langle 111\rangle$-oriented Si planes. The etching process continues with an inward slope angle of $54.7^{\circ}$ until the passivation layer on the front side of the wafer is reached. Images looking inside the truncated pyramids from the backside are shown in Figure 2.

The size of the suspended membrane at the frontside of the wafer determines the active area where cells may be contacted via microfluidics from below. For cell experiments, we prepared active areas of approximately $1-900 \mu \mathrm{m}^{2}$.

To integrate nanoporous structures with the microfluidics, a thin aluminum film of $500 \mathrm{~nm}$ was deposited onto the silicon substrate using sputtering or electron beam evaporation at a deposition rate of 0.7 and $1 \mathrm{~nm} / \mathrm{s}$, respectively. Herein, two different approaches were realized. On one hand, the aluminum was deposited on the front side of the wafer (frontside approach, see Toh et al. ${ }^{8}$ ). On the other hand, the 


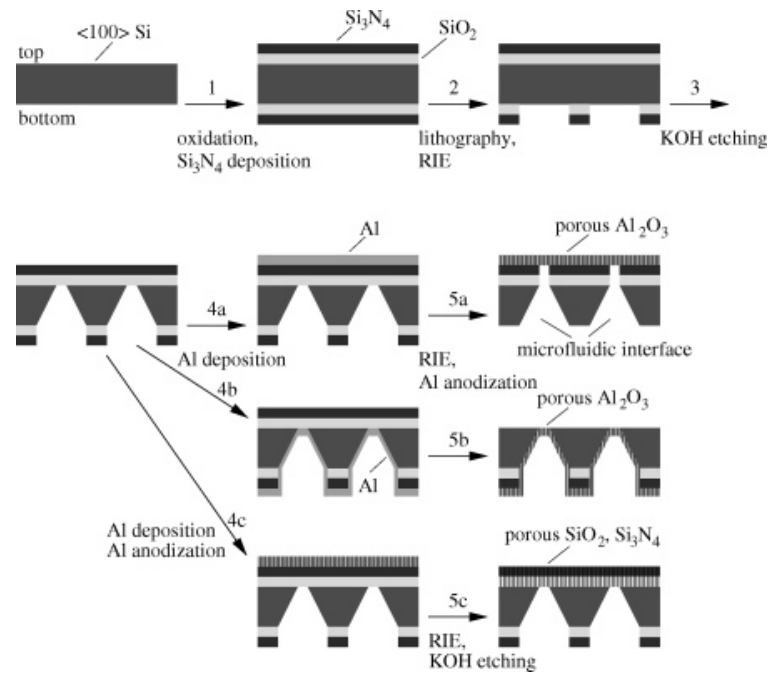

Figure 1. Schematic of the device fabrication processes. Doublesided polished wafers are passivated with $\mathrm{SiO}_{2} / \mathrm{Si}_{3} \mathrm{~N}_{4}$ (1). The bottom side of the wafer is subsequently structured with lithography, reactive ion etching (RIE) (2), and anisotropic etching in $20 \% \mathrm{KOH}$ solution (3). To prepare suspended porous alumina membranes, $\mathrm{Al}$ is deposited on the top (4a) or bottom (4b) side of the chip and anodized after removal of the $\mathrm{SiO}_{2} / \mathrm{Si}_{3} \mathrm{~N}_{4}$ passivation layer (5a, 5b). For the creation of porous $\mathrm{SiO}_{2} / \mathrm{Si}_{3} \mathrm{~N}_{4}$ membranes, the aluminum layer is anodized directly after $\mathrm{Al}$ deposition (4c). The nanoporous alumina structure is then transferred onto the passivation layer by $\mathrm{RIE}$, and the remaining porous alumina layer is removed in $\mathrm{KOH}$ solution.
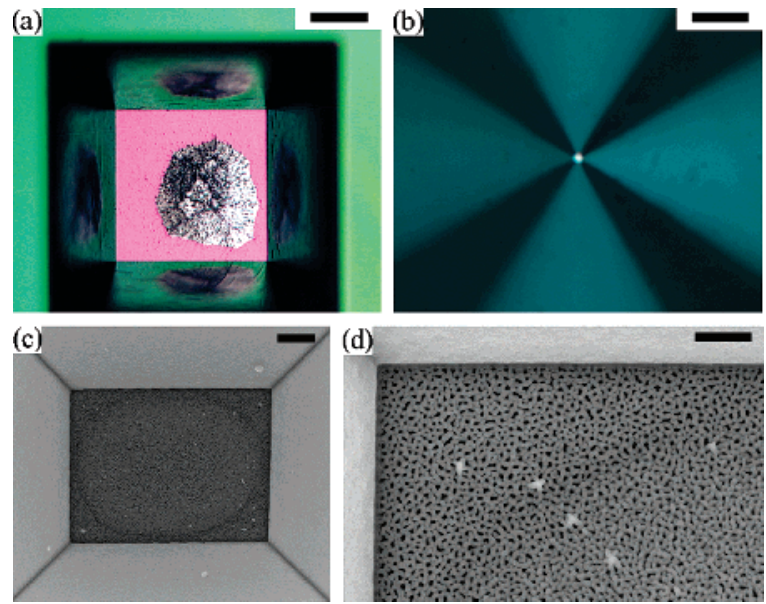

Figure 2. Light microscopy and SEM images of the truncated pyramids etched into the silicon wafers: Image a is taken just before etching is completed. A patch of silicon is therefore remaining on the $\mathrm{SiO}_{2} / \mathrm{Si}_{3} \mathrm{~N}_{4}$ passivation membrane. The smooth side walls show reflections of the silicon patch in the center. Image b shows a much smaller structure, the area of the suspended film being on the order of $\approx 1 \mu \mathrm{m}^{2}$. Aluminum has already been deposited on the top surface of the wafer, thereby enhancing the reflection at the bottom of the etched structure. SEM images $\mathrm{c}$ and d depict the nanoporous alumina structure of a suspended membrane patch. The scale bars are $200,10,4$, and $1 \mu \mathrm{m}$ for images a, b, c, and d, respectively.

aluminum was deposited from the backside of the wafer through the apertures of the microfluidic structures (backside approach). The second approach results in small patches of aluminum/alumina on the front side of the wafer. In addition it facilitates the integration of electronic circuits on the front side of the wafer, which will not interfere with the prepara-

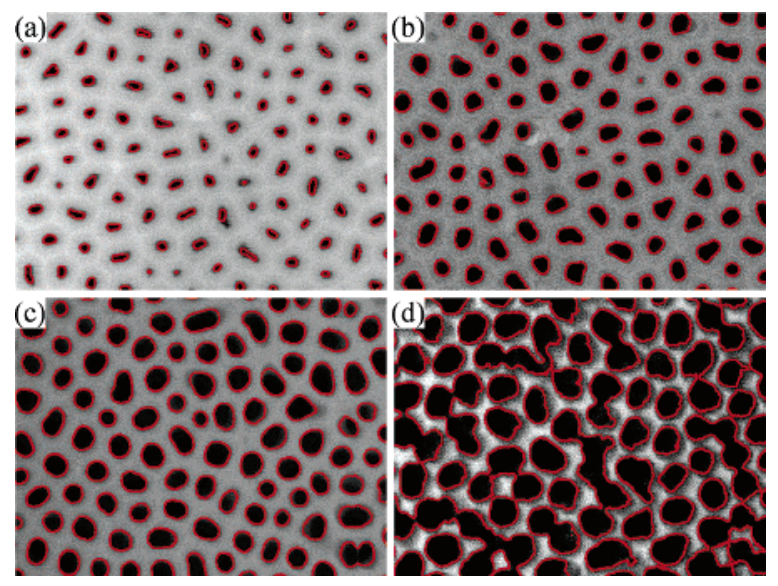

Figure 3. SEM images of nanoporous surfaces at the bottom of the truncated silicon pyramids after pore enlargement in 5\% $\mathrm{H}_{3} \mathrm{PO}_{4}$ for (a) 0 , (b) 10, (c) 40, and (d) $70 \mathrm{~min}$. The width of each image is $1 \mu \mathrm{m}$.

tion process of the nanoporous membranes. In both approaches the silicon nitride and remaining silicon oxide were subsequently etched with RIE $\left(\mathrm{CF}_{4}, 20 \mathrm{~mL} / \mathrm{min} ; \mathrm{CHF}_{3}, 20\right.$ $\mathrm{mL} / \mathrm{min}$ ) at 0.03 mbar and a power of $300 \mathrm{~W}$. This step exposes the bare aluminum for subsequent anodization. Either the complete or only the suspended aluminum film is anodized using sulfuric $(3 \%, 25 \mathrm{~V})$, oxalic $(0.3 \mathrm{M}, 40-65$ $\mathrm{V})$, or phosphoric $(5 \%, 60-120 \mathrm{~V})$ acid. Applied voltage and etching solutions depend on the desired interpore distance. Alumina barrier layer removal and pore widening were conducted in $5 \%$ phosphoric acid at $20{ }^{\circ} \mathrm{C}$ for 5 to 70 min depending on the desired pore width of the alumina membranes. Figure 3 shows scanning electron microscopy (SEM) images of a suspended porous alumina membrane with increasing pore diameters on a microfluidic structure.

Porosity parameters, e.g., average pore size and interpore distance were obtained by image processing methods based on threshold separation. In contrast to the fabrication of thick porous alumina foils, which exhibit highly hexagonally ordered pores of large aspect ratios, ${ }^{9-11}$ anodization of thin aluminum films deposited on a substrate leads to an increased variance of the pore parameter in the nanoporous alumina film. A possible explanation of the larger variance of the pore parameters is the shorter etching time determined by the thickness of the aluminum film. However, similar to the fabrication process of highly ordered nanoporous alumina films the average interpore distance is determined by the applied voltage during anodization. The ratio of average interpore distance to anodization voltage was 2 and $1.3 \mathrm{~nm} / \mathrm{V}$ for sputtered and e-beam evaporated aluminum films, respectively. Manipulation of interpore distance in combination with subsequent pore enlargement enabled us to control porosity parameters of the cell-substrate interface.

For the fabrication of porous silicon nitride/silicon oxide membranes the aluminum was directly anodized on top of the nitride layer. The pattern of the nanoporous alumina was transferred onto the underlying substrate by a two-step RIE process (Ar, $40 \mathrm{~mL} / \mathrm{min}, 0.02$ mbar, $300 \mathrm{~W} ; \mathrm{CF}_{4}, 20$ $\mathrm{mL} / \mathrm{min}$, and $\mathrm{CHF}_{3}, 20 \mathrm{~mL} / \mathrm{min}, 0.03 \mathrm{mbar}, 300 \mathrm{~W}$ ). Prior to reactive ion etching the porous alumina film was thinned 


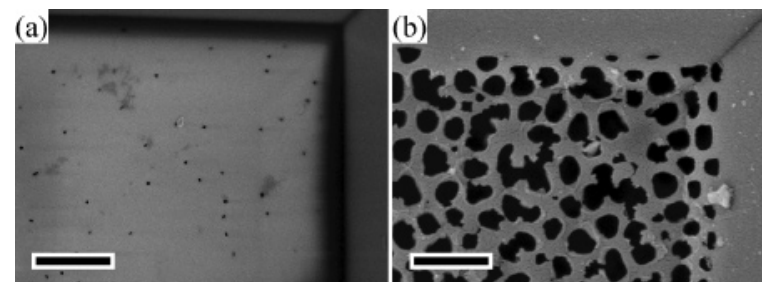

Figure 4. SEM images of suspended silicon oxide/silicon nitride membranes. Image a shows a porous membrane with a sparse distribution of pores (porosity $<0.2 \%$ ). The membrane in image $b$ is strongly perforated resembling closely the alumina template (porosity $\approx 44 \%$ ). Scale bars are 500 and $300 \mathrm{~nm}$, respectively.

in chromic acid $\left(45 \mathrm{~g} / \mathrm{L}\right.$ in $\left.3.5 \% \mathrm{H}_{3} \mathrm{PO}_{4}\right)$ to enhance effectiveness of the subsequent RIE process. Depending on etching time the nanopores of the alumina template were either completely or only partially transferred onto the underlying silicon nitride/oxide membrane. In Figure 4, different suspended porous silicon nitride/oxide membranes resulting from similar alumina templates are compared. The porosity of the membrane will affect the molecular transport of the system.

To enhance wettability of the microfluidic structures, the chips are made hydrophilic in an oxygen plasma (200 W, 1 mbar) for $2 \mathrm{~min}$.

To study biocompatibility and to investigate the local delivery of chemicals, we performed cell experiments on the suspended nanoporous membranes. Before cell plating, the chips were sterilized in 70\% ethanol. The cells were plated on the top of the chip, while the backside was connected to a microfluidic chamber. We examined one cell line of the human embryonic kidney (HEK293) and two primary neuronal cell types of the cortex of rat embryos and of the locust thoracic ganglia. HEK293 cells are genetically engineered to express a voltage-gated potassium ion channel and have already been used as a model cell line in electrophysiological experiments. ${ }^{12}$ For the preparation of primary neurons, the cortices of embryonic Wistar rats (18 days gestation) and the thoracic ganglia of locusts were isolated and dissociated following standard procedures. ${ }^{13,14}$ HEK293, rat, and locust cells were plated at densities of $10000 / \mathrm{cm}^{2}$ $16000 / \mathrm{cm}^{2}$, and $200 / \mathrm{cm}^{2}$, respectively. Adhesion studies were performed on the bare substrates as well as on substrates coated with polylysine (PLL), extracellular matrix gel (ECM), and concavalinA (conA). Both HEK293 cells and embryonic rat neurons were maintained at $37^{\circ} \mathrm{C}$ in $5 \% \mathrm{CO}_{2}$ while the insect neurons were maintained in air at $29{ }^{\circ} \mathrm{C}$.

Cell growth and adhesion to the substrate were monitored up to 14 days after cell plating. Also, the electrophysiological properties of cells were investigated using standard patch clamp techniques. ${ }^{15}$ However, no dependence of electrophysiological properties of cells on the underlying substrate was found.

SEM was performed to visualize the adhesion of cells on the nanoporous substrates. The cells were prepared for SEM using glutaraldehyde fixation followed by critical point drying. They were incubated for $8 \mathrm{~h}$ in a $\approx 3 \%$ glutaraldehyde solution of the same osmolarity as the culture medium.

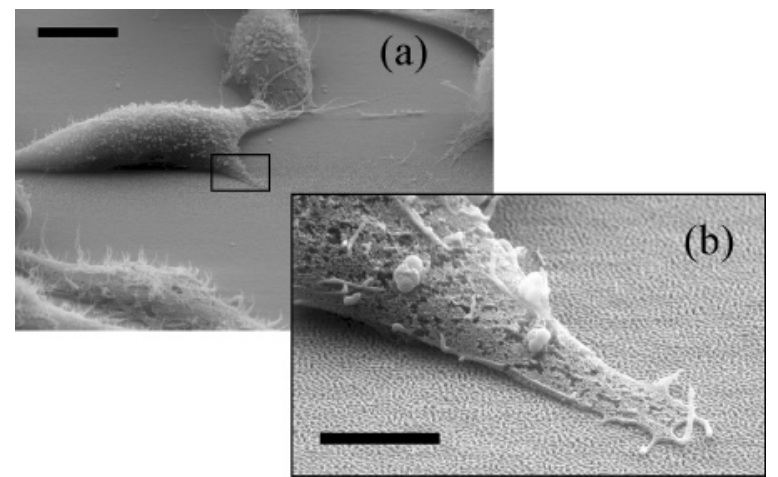

Figure 5. SEM image of HEK cells growing on a porous alumina substrate. (b) is an image enlargement of (a) showing the nanopores. The scale bars for (a) and (b) are 10 and $2 \mu \mathrm{m}$, respectively.

Afterward, the solution was exchanged stepwise with 2-propanol. Subsequently, chips were placed in a critical point dryer (Bal-TecCPD030), where the 2-propanol was exchanged by carbon dioxide followed by critical point drying. Before SEM imaging, a thin gold film was sputtered onto the dried samples. In Figure 5 an example of HEK293 cells growing on porous alumina is shown. The cells adhere nicely to the substrate, but no filaments seem to grow into the nanopores themselves. The latter might occur if the pore size of the substrate is increased. We tested porous substrates with average interpore distances from 30 to $200 \mathrm{~nm}$ and porosities up to $40 \%$. All substrates tested were biocompatible in the sense that the cells were able to adhere to and grow on the surface. Both neuronal cell types also showed outgrowth of neurites. However, the rat neurons showed better adhesion and outgrowth of neurites on substrates coated with ECM to facilitate adhesion. The same coating was necessary to facilitate growth of the rat neurons on silicon oxide/silicon nitride surfaces. For both neuronal cell types it was crucial to avoid any contact of the cell medium to remaining unanodized aluminum film. Contact to the aluminum film leads to cell detachment and cell death of the neurons after a few days in culture. The alumina as well as the silicon nitride membranes were observed to be stable for the duration of the experiments (up to 14 days of cell culture). SEM imaging and secondary cell experiments showed that porous silicon nitride membranes could be reused after cleaning in piranha solution. However, no cleaning procedure ensuring the intactness of porous alumina membranes for further reuse could be found.

The viability of cells was tested using an ethidium bromide/acridine orange staining procedure in PBS at 5 and $1.5 \mu \mathrm{g} / \mathrm{mL}$, respectively. Damaged cells appear orange due to the intercalation of ethidium bromide (EB) into their DNA and RNA. Cells with intact membranes are stained green by the membrane-permeant dye acridine orange (AO). The same dye at five times the concentration was used to observe local delivery of chemicals through the nanoporous membranes. Therefore the dye solution was applied to the backside of the chips. Cells growing on the top surface were monitored in a fluorescence microscope for up to $1 \mathrm{~h}$ after chemical delivery. The cells were only exposed to light during image acquisition to avoid unnecessary bleaching. Figure 6 depicts 


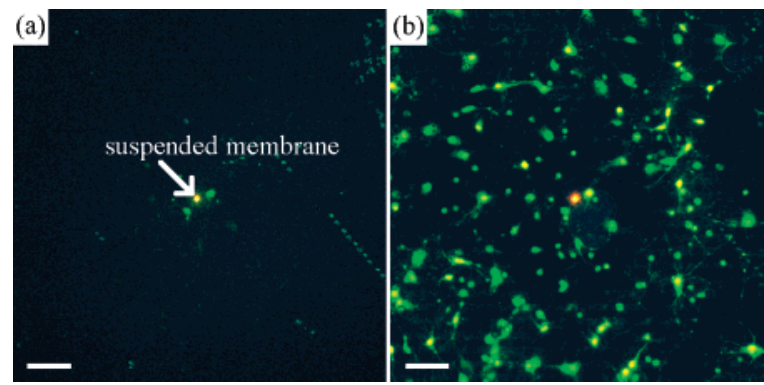

Figure 6. Fluorescence image of rat neurons (7 days in culture) growing on porous alumina (average pore diameter $54 \pm 13 \mathrm{~nm}$, porosity $\approx 30 \%$ ). Image a was taken 20 min after AO exposure from the backside of the chip. Image $b$ shows all the cells growing on the substrate after AO staining from the front side. The chip is illuminated from the backside to reveal the position of the porous suspended alumina membrane (area $140 \mu \mathrm{m}^{2}$ ). Scale bars are $100 \mu \mathrm{m}$.

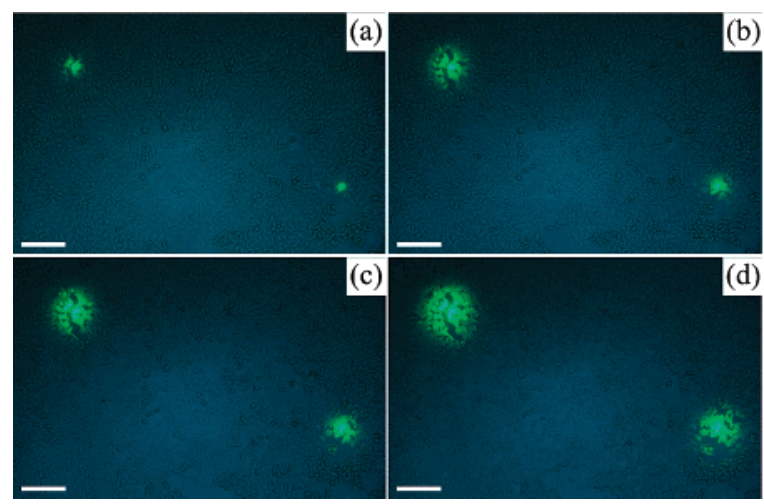

Figure 7. Fluorescence image of HEK cells ( 3 days in culture) on a chip with open apertures taken $1,5,15$, and 25 min after AO application from the backside of the chip. The area of a single aperture is $80 \mu \mathrm{m}^{2}$. The scale bars are $100 \mu \mathrm{m}$.

rat neurons growing on porous alumina with a porosity of approximately $30 \%$.

In addition to the front light fluorescence, the backside of the chip was illuminated to indicate the position of the suspended nanoporous alumina membrane. Image a was taken 20 min after application of acridine orange to the backside of the wafer. Only cells in the vicinity of the active area are stained by the DNA intercalating dye AO. To reveal all the cells growing on the surface, AO was subsequently applied to the front side of the chip (see Figure 6b).

The area at which cells are affected by the dye depends on the time as well as the size of the active area and its porosity. For comparison, we investigated the staining of HEK cells growing on chips with completely opened apertures (100\% porosity, Figure 7) and apertures covered with sparsely perforated silicon nitride membranes (Figure 8).

In the former it can be seen how cells adjacent to the aperture are affected over time. To reduce convective effects, which might occur during application of the dye, the front side of the chip was covered by a planar transparent polystyrol surface.

For the chips with sparsely perforated silicon nitride membranes, convection from backside to frontside is greatly reduced due to the high flow impedance in the nanopores.

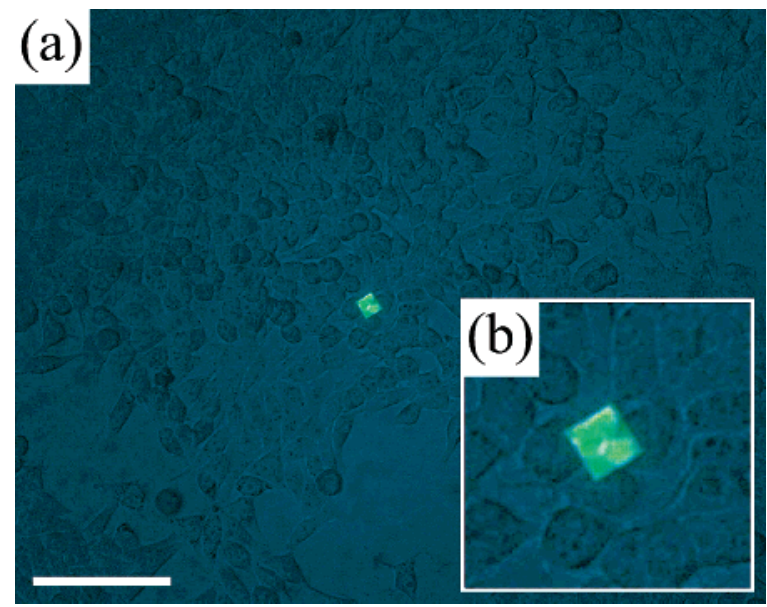

Figure 8. Fluorescence image of HEK cells (3 days in culture) on a chip with silicon nitride membranes $\left(<0.2 \%\right.$ porosity, $200 \mu \mathrm{m}^{2}$ area) 15 min after $\mathrm{AO}$ application from the backside of the chip. The scale bar is $100 \mu \mathrm{m}$. Image $\mathrm{b}$ is a $3 \times$ enlarged image section of image a.

Similar to the experiments with membranes of high porosity, initial staining of cells can be seen a few seconds after application of the dye. However, in the case of sparsely perforated membranes as shown in Figure 8, only the parts of the cells growing directly on the porous membrane were observed to yield any fluorescence, even several minutes after dye application. This suggests that small area membranes with low porosities are best suited to locally address single cells or even subcellular regions. Active areas with high porosities facilitate larger transport rates across the porous membrane. They might be better suited to perform experiments in which weaker chemical gradients are required. For example, on-chip chemically guided cell growth requires larger areas of the chip surface to be affected by the chemical stimuli. An important factor, which can influence molecular transport in nanopores is the clogging of pores due to biofouling. ${ }^{2}$ It can be expected that biofouling partially inhibits or decreases the transport rates across the membranes, especially during long-term cell culture studies. Popat et al. showed that biofouling of alumina membranes during biofiltration experiments can be effectively diminished by applying poly(ethylene glycol) (PEG) coatings. ${ }^{16}$ The development of antifouling coatings for cell-based nanoporous interfaces might contribute to the development of chemical cell communication systems with stable transfer rates. However, a challenge will be to develop antifouling methods, which do not interfere with cell adhesion. For example, the neuronal cells used in these studies do not grow on PEGcoated substrates. A coating that is confined to the inside of nanopores might be promising in such an approach.

In conclusion, we presented a biohybrid system based on suspended nanoporous alumina or nanoporous silicon oxide/ nitride membranes on a silicon chip. The nanoporous membranes act as a cell interface and facilitate control of the cell environment with minute quantities of chemicals. It was shown that cells can be addressed highly localized through the nanoporous membrane patches. The on-chip setup allows for the integration of other stimulation or 
sensing devices, such as microelectrode arrays, providing an opportunity to combine high-resolution electrical and chemical interfaces on a single chip.

Acknowledgment. We thank R. Helpenstein and S. Weigel for help with the cell culture. Also we thank B. Hermanns, H. Kohlstedt, and A. Steffen for support with the clean room facilities. HEK293 cells were provided by the Institute of Biological Information Processing of the Research Center Juelich.

\section{References}

(1) Peterman M. C.; Mehenti, N. Z.; Bilbao, K. V.; Lee, C. J.; Leng, T.; Noolandi, J.; Bent, S. F.; Blumenkranz, M. S.; Fishman, H. A. Artif. Organs 2003, 27, 975.

(2) Desai, T. A.; Hansoford, D. J.; Kulinsky, L.; Nashat, A. H.; Rasi, G.; Tu, J.; Wang, Y.; Zhang, M.; Ferrari, M. Biomed. Microdevices 1999, 2, 11

(3) Karlsson, M.; Pålsgård, E.; Wilshaw, P. R.; Silvio, L. D. Biomaterials 2003, 24, 3039.

(4) Takoh, K.; Takahashi, A.; Matsue, T.; Nishizawa, M. Anal. Chim. Acta 2004, 522, 45.
(5) Crouse, D.; Lo, Y.-H.; Miller, A. E.; Crouse, M. Appl. Phys. Lett. 2000, 76, 49.

(6) Sander, M. S.; Tan, L.-S. Adv. Funct. Mater. 2003, 13, 393.

(7) Rabin, O.; Herz, P. R.; Lin, Y.-M.; Akinwande, A. I.; Cronin, S. B.; Dresselhaus, M. S. Adv. Funct. Mater. 2003, 13, 631.

(8) Toh, C.-S.; Kayes, B. M.; Nemanick, E. J.; Lewis, N. S. Nano Lett. 2004, 4, 767.

(9) Jessensky, O.; Müller, F.; Gösele, U. Appl. Phys. Lett. 1998, 72, 1173

(10) Masuda, H.; Yamada, H.; Satoh, M.; Asoh, H.; Nakao, M.; Tamamura, T. Appl. Phys. Lett. 1997, 71, 2770.

(11) Nielsch, K.; Choi, J.; Schwirn, K.; Wehrspohn, R. B.; Gösele, U. Nano Lett. 2002, 2, 677.

(12) Straub, B.; Meyer, E.; Fromherz, P. Nat. Biotechnol. 2001, 19, 121.

(13) Vogt, A. K.; Stefani, F. D.; Best, A.; Nelles, G.; Yasuda, A.; Knoll, W.; Offenhäusser, A. J. Neurosci. Methods 2004, 134, 191.

(14) Mourzina, Y.; Steffen, A.; Kaliaguine, D.; Wolfrum, B.; Schulte, P.; Böcker-Meffert, S.; Offenhäusser, A. J. R. Soc. Interface DOI: 10.1098/rsif.2005.0099.

(15) Hamill, O. P.; Marty, A.; Neher, E.; Sakmann, B.; Sigworth, F. J. Pflügers Arch.-Eur. J. Physiol. 1981, 391, 85.

(16) Popat, K. C.; Mor, G.; Grimes, C.; Desai, T. A. J. Membr. Sci. 2004, 243, 97.

NL052370X 